\title{
Automatic Generation of Bond Graph Models of Process Plants
}

\author{
Sebastian Beez \\ Helmut-Schmidt-University \\ Holstenhofweg 85 \\ 22043 Hamburg, Germany \\ sebastian.beez@hsu-hh.de
}

\author{
Alexander Fay \\ Helmut-Schmidt-University \\ Holstenhofweg 85 \\ 22043 Hamburg, Germany \\ alexander.fay@hsu-hh.de
}

\author{
Nina Thornhill \\ Imperial College London \\ South Kensington Campus \\ London SW7 2AZ, UK \\ n.thornhill@imperial.ac.uk
}

\begin{abstract}
This paper presents an application for the automatic generation of Bond Graph models.

The basis for this automated creation is a modified plant model in the XML-format according to the IEC PAS 62424 (CAEX). The application developed in the programming language $C$, extracts and converts the information which is, then, stored in a model file meeting the requirements and structure of the modelling/simulation-language Dymola. Bond Graphs are used as the modelling technique since they do not distinguish between different energy domains and, therefore, combine several advantages against other modelling techniques.

The developed application can be used for multiple purposes such as simulations, visualizations and other specific tasks that might emerge during the planning and operation process of plants and other engineering systems.
\end{abstract}

\section{Introduction}

Nowadays system models are used for various kinds of simulations and research and the profit gained from such models/ simulations is essential throughout the different engineering steps of building, testing and running all kinds of systems. The models therefore need to act like their real counterparts in terms of continuous behaviour. Especially if they represent a chemical process that involves several media these models should include the physics and laws of hydrodynamics, reaction chemistry and heat- and mass-transfer.

In general, creating models by hand is timeconsuming and, therefore, costs a lot of money. That is why an automatic creation of such models from existing plant data is a welcome and desirable alternative. Object-oriented Computer Aided Engineering tools offer the potential for such an automatic creation.
Object-oriented representations of plants and processes are becoming more available and are a convenient way to store and exchange information such as process diagrams between different engineering domains. One way to do this is the Computer Aided Engineering Exchange-format, CAEX. Bond Graphs on the other hand are a modelling method to describe the power flow within a system. Bond Graphs allow the representation of hybrid systems more easily than other modelling techniques since they do not differentiate between mechanical, thermal, hydraulic or other energy domain systems.

The work this paper is based on was carried out in two projects. The main aim in the first project was to provide an application which is able to automatically generate Bond Graph models out of CAEX files. After the first development stage of the application was finished, the second project's aim was to broaden the abilities of the program and overcome limitations such as elements lacking sensor- and actuator-interfaces and multiple inflows and outflows. Furthermore, more options for the user to influence the model had to be implemented.

After this introduction into the topic, the second chapter provides all the background knowledge needed to understand the subsequent chapters. A brief presentation of the tools and their common properties, the preparatory work on the necessary data files and the methods for the conversion are presented to the reader. A validation chapter gives proof of the abilities of the application and shows possible ways of using the automatically generated models. A conclusive part finalizes this paper by summarizing the achievements and providing a future outlook.

\section{CAEX and Bond Graphs}

This section is to introduce the basis of this paper. The Computer Aided Engineering Exchange-format (CAEX), Bond Graphs, the modeling software Dymola and their common properties will be presented in the following sections. 


\subsection{CAEX}

The CAEX format was developed by the Chair of Process Control Engineering of RTWH Aachen and ABB Research Centre. CAEX and other languages/ schemas are based on the Extensible Markup Language (XML), which is a standard way to describe data. XML was initially developed to share data across the internet [9]. The CAEX schema functions as a vendorindependent neutral data exchange format between plant engineering tools. It was developed to reduce the amount of costs and time during plant design. CAEX represents the structural and functional behaviour of a plant. Thus, CAEX models are meta-models which describe elements in an abstract way and with an arbitrary number of attributes. They contain the information stored in Piping and Instrumentation Diagrams (P\&ID) as well as additional information. The possibility to extend models is another important feature. CAEX files are used during plant design thus they enable different groups to add information and pass the file on to the next group. CAEX methodology provides this feature. The models can be extended and completed anytime without a need to have a new agreement of the structure.

Similar to XML, CAEX files have a schema, too. It prescribes the structure of a CAEX file. At the beginning of a document there is a declaration. Thereafter the root node begins. Besides a header, a CAEX root node has four major child notes. The SystemHierarchy gives an overview of the whole system and all the elements including all the links between the elements. However, the SystemHierarchy provides no further information about the elements. The RoleClassLibrary lists all possible roles of elements. It is comparable to the P\&ID. Only a symbol is used to describe a certain task, such as tank, valve, pump and the like. The third major child node is the SystemUnitClassLibrary. This library contains special information about elements. It can present the internal structure of an element as well as specifications and operating parameters. This child node allows integration of different libraries. For example, companies can use it to supply a portfolio of their products. The fourth child node is the InterfaceClassLibrary, which describes different interfaces and their characteristics.

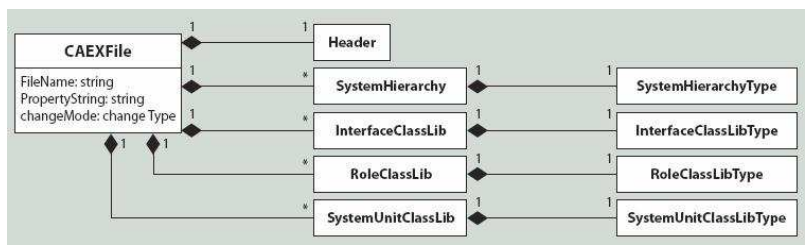

Figure 1: Structure of a CAEX file (from Drath et al. (2005))
Figure 1 presented the CAEX schema used during the projects.

Drath et al. (2005) provides further information on the structure and the relations between these major parts of the CAEX file.

\subsection{Bond Graphs and Dymola}

A Bond Graph is a modelling methodology that was developed by Professor Henry M. Paynter. The main idea is to model the power flow through a system assuming energy conservation. This methodology has several advantages. It is easier to model hybrid systems since multi-energy domain models can be created without a use for own models for every energy domain. A second advantage is bi-directionality of the elements. If elements produce any kind of effect that influences upstream elements there is no need to model a feedback loop. This effect will propagate backwards through the upstream elements anyway.

The power flow is modelled using two variables, effort, $e$, and flow, $f$, which yield power if multiplied. Therefore they are called power-variables. Furthermore there are momentum, $p$, and displacement, $q$, which yield energy if multiplied. The relations between all these variables are explained in more detail in Karnopp et al. (1990) and Cellier (1991).

With the help of these variables a range of elements was created. The developer was an electrical engineer, thus, most of the elements that were used for creating models have synonyms used in this particular area. Basic components which appear in every system can be modelled using the same variables and equations.

- Resistors, capacities, inductances

- Transformers and gyrators

- Effort and flow sources

- $\quad$ Parallel and series-junctions

appear in every system, energy domain independent. Only the units are changing. That is why hybrid systems are easier to handle with the Bond Graph methodology. Further information related to Bond Graphs can be found in Karnopp et al. (1990)and Cellier (1991).

Dymola was the modelling/simulation software for the projects. It is based on Modelica which is an equation-based modelling language. Dymola enables modelling via Drag\&Drop and creates the Modelica code behind the graphical models itself. There are plenty of freeware libraries for Dymola for various kinds of engineering areas, a Bond Graph library amongst them. The Bond Graphs library includes a well-equipped set of basic elements and some special sub-libraries. Besides modelling, simulations can be run with Dymola. The software includes several 
solving algorithms and certain possibilities to influence the simulation. Furthermore generated models can be exported and used in other simulation environments such as MatLab/Simulink.

For further information about Dymola itself or the use of this software, see Fritzson (2004) or the Dymola User Manual provided along with the program. The following figure shows the Dymola window and a model composed of Bond Graph elements.

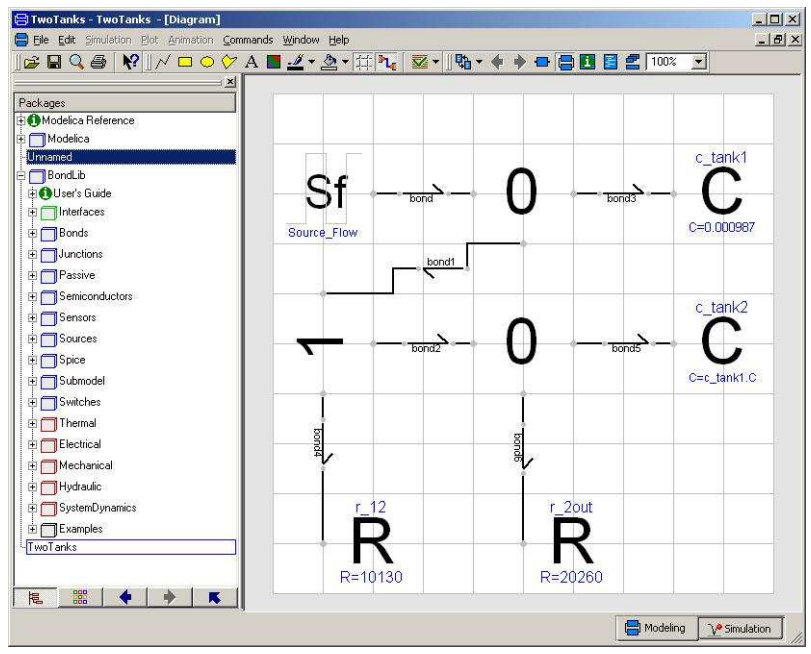

Figure 2: Dymola User Interface with Bond Graph model

\section{Generation methodology}

\subsection{Common properties}

There are several similarities between CAEX files and Bond Graph models in Dymola; especially according to the structure. These similarities are most helpful throughout an automatic conversion.

As mentioned in 2.1 CAEX the SystemHierarchy lists all elements appearing in the system. This is beneficial since a list of all the elements can be generated and then worked off. Utilizing this, a complete list of all the elements can be written into the model file. The links between these elements, also given in the SystemHierarchy, make up the second part of the model file. Hence, they have to be transferred as well. The SystemUnitClassLibrary contains special information about each element. This is very advantageous for specifying each element and equipping it with its own properties. Since Dymola is working with different libraries the RoleClassLibrary in a CAEX file is a convenient place to store the Dymola-library path of the corresponding element.

The CAEX requirements presented in the following section presents how these structural similarities can be used creating the CAEX file from which the model is built.

\subsection{CAEX requirements}

The automatic generation of a Bond Graph model demands information about all the elements. These demands especially apply to the CAEX file that the model is derived from. The following section points out the conditions the CAEX file has to meet in order for the conversion to function properly.

In more detail, the SystemHierarchy stores every single element along with its individual references to RoleClassLibrary and SystemUnitClassLibrary. Furthermore the physical links to other elements are listed. Since some elements have signal connections, these links are stored in this section as well.

Following the reference in the SystemHierarchy, the RoleClassLibrary provides the matching Bond Graph element together with the path in the Bond Graph library. That is due to the focus of this project, which was not to scan this library for matching elements.

The SystemUnitClassLibrary has to provide the parameters for the matching Bond Graph elements. These parameters can either be abstract details like a capacity according to a pressure if the component is a basic Bond Graph element or, like in common CAEX files, geometric details of the elements, such as height and cross-section area if the model is one of the components developed during the second project.

These requirements account for another important aspect. The level of detail in the Dymola model is determined by the level of detail provided in the CAEX file. Thus, the more information about an element is given in the CAEX file, the more detailed is the model and the simulation result from this component.

\subsection{Approach for the automatic generation}

The generation methodology utilizes the common properties in structure and the restriction put on the content of the CAEX file.

The method of converting a CAEX file into a model is divided into three parts. The first part comprises collecting all the information from the CAEX file. The fact that the SystemHierarchy contains all the elements of the system makes it a convenient point to start with. A list of all the elements is created first and then filled with information about each component starting with its name. The data about the links each element has to the others is then taken from the CAEX file and added to this list. Thereafter the references to its role and to its specific and detailed information are tracked and these information are written into the list as well. This finalizes the first step of gathering all the information and bundling it in a list. The following figures show a CAEX file and its structure. They present an example of two tanks and show how the information about these tanks is structured. 


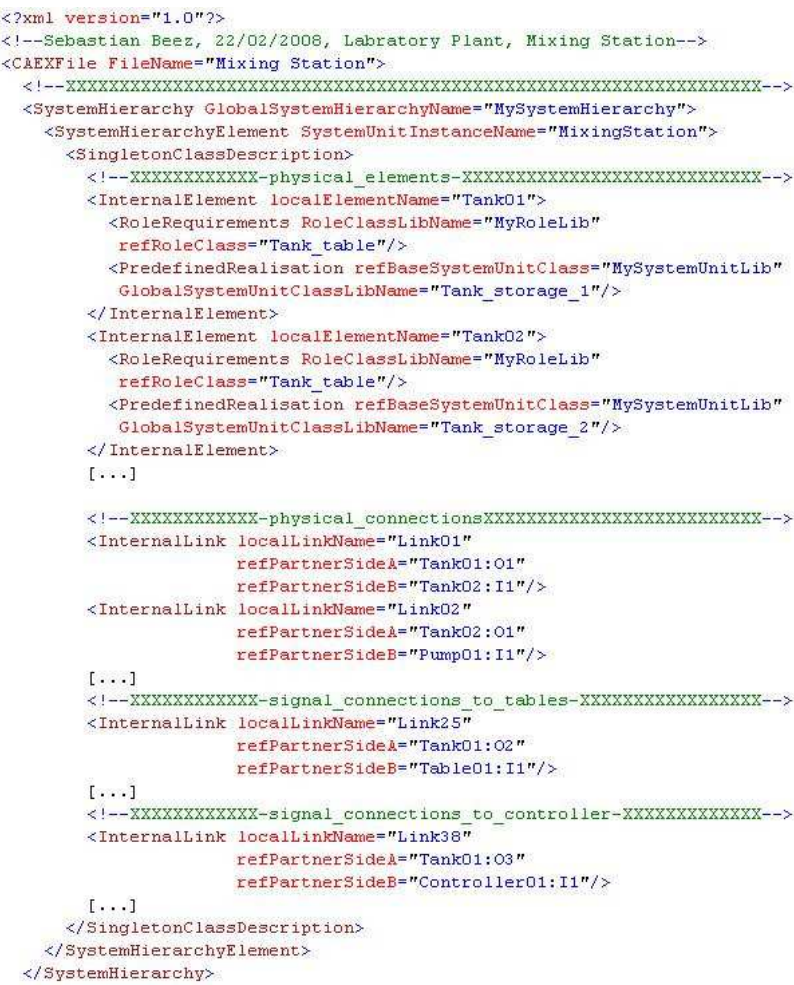

Figure 3: SystemHierarchy of a CAEX file

The referenced RoleClasses and the SystemUnitClasses are presented in the Figure 4.

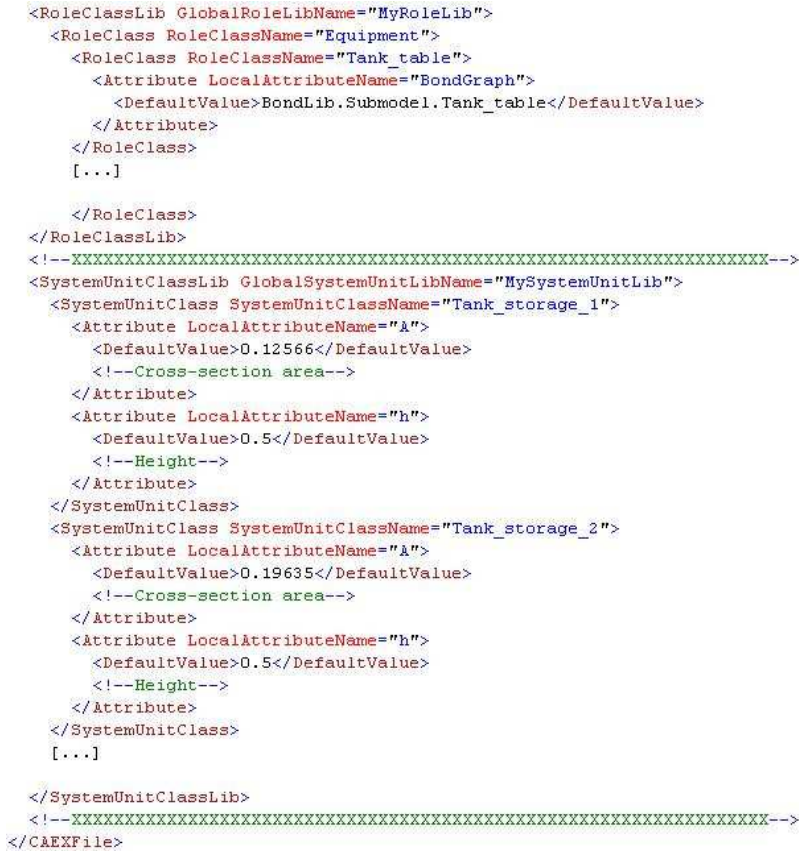

Figure 4: RoleClass- and SystemUnitClassLibrary of a CAEX file
After collecting all the information from the CAEX file it needs to be adapted to the demands of the modeling software. This means that the second step in the developed methodology is reordering the gathered information according to the structure of a single entry in the model file and considering the overall structure of the model file.

Finally, the third part of the generation methodology is to write the model file. After a header and an annotation necessary for the simulation in Dymola all the components of the model are listed. After the components and the keyword "equation" all the links need to be written into the file. An end-section finished the model file that looks like the following figure for the example of the two tanks.

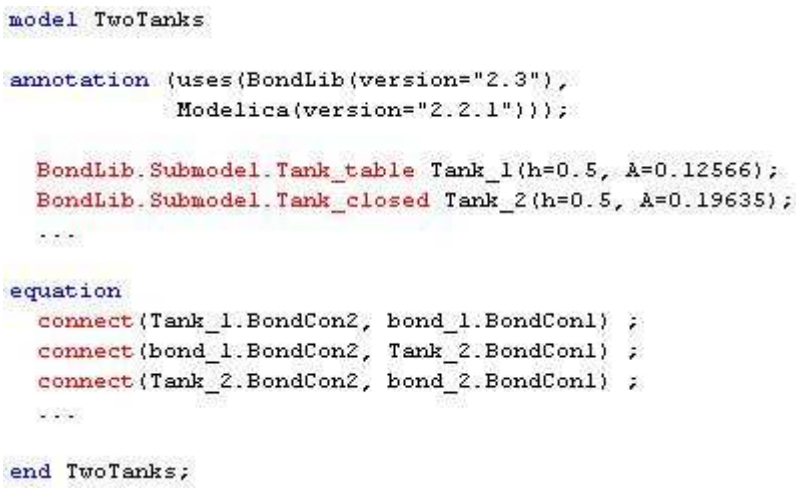

Figure 5: Model file in Dymola

\section{Application of the methodology}

The aim of this section is to explain how the developed application utilizes the approach chosen for the model generation. The three steps of the methodology are maintained in general and will be explained in more detail in the following lines.

The user determines the behaviour of the program. Hence, the first part entails the user choosing a CAEX file after having started the application. The second part takes place without any user interaction. The data from the chosen file is read and parsed into the application. Every InternalElement in the SystemHierarchy is allocated to an object. Not only is the element name itself stored in this object, but also its references and details. Finalizing the second part of the generation methodology, the object is then added to an elementList.

The Element-object and its attributes, where all the data for a single component is stored in, is depicted in the following figure. Thereafter the first step of the generation methodology is summarized graphically. 


\begin{tabular}{l} 
Element \\
\hline - Name \\
- Number \\
- Bond Graph representation \\
- Specifications \\
- Links to \\
- Links from \\
- Position X \\
- Position Y
\end{tabular}

Figure 6: Element-object and attributes

1. The User chooses a CAEX file

2. For each element in the file the following steps are performed:

- Create an "Element"-object

- Save name from SystemHierarchy

- Add a number

- Save path in the Dymola library from the referenced RoleClass

- Save specifications from the referenced SystemUnitClass

- Save the connections from the SystemHierarchy

- Add an x- and an y-position

- Add the "Element"-object to the elementList

\section{Figure 7: Procedure of reading the CAEX file}

The position-attributes are necessary for positioning each component in the Dymola user interface. To proceed with the generation of the model file further user input is required. The user needs to provide a model name and file path to store the model file to. In addition, the user can manipulate the model by giving parameters for the simulation. As an example, the user can set the level of medium inside a tank to a certain value at the beginning of the simulation. These adjustments are added to the specification of the pertaining element.

Step two is the biggest and most important step. It is subdivided into two steps.

The first sub-step is reordering the attributes of each element to meat the required structure for a single component. In the case of Dymola as the modeling language the single entry for a tank as shown in Figure 3 would then consist of the path in the Dymola library (as given in the RoleClass), the name (as stated in the SystemHierarchy) and the details (provided by the SystemUnitClass). Every element from the first list and its details is reordered like that and saved in a second list. With respect to the overall structure of the Dymola model only the reordered elements are included in this second list, called entryList.

Before building the model file some of the elements need further preparation. Since Bond Graphs are the modelling technique, some elements such as resistors, $R$, and capacitors, $C$, which are basic elements, need to be attached to junctions in order to work. These junctions and the bonds to connect elements and junctions are also added to the entryList. The code for the connections between the passive elements and the bonds as well as between the bonds and the junctions is stored in the third list, called connectList.

The second sub-step is to link all the elements according to the information gained from the CAEX file. The information about the links between the elements is taken from the first list, reordered and written into the connectList. The following figure summarizes step two.

1. For each object in the elementList the following steps are performed:

- The details are reordered and added to the entryList

- If necessary, bonds and junctions are added to the entryList

- Connections between the component, bond and junction are added to the connectList

- Connections between the components are written into the connectList

\section{Figure 8: Preparing and linking the elements}

Finally, the model file itself is created. This starts with opening a new file and writing the header in. It consists of the keyword model, the name (provided by the user via the Graphical User Interface) and some annotations for Dymola to compile the model. After the header section, all the elements are listed. After completing the entryList in the last step, each entry is written into the model file. Then the keyword equation followed by the content of the connectList is added to the model file. Finalizing this step, the keyword end and the model name are written to the model file. Thereafter the file is saved, shut and looks like Figure 5: Model file in Dymola. Figure 9 summarizes the final part of the model generation if Dymola is the modelling language. 
1. Open a model file to write in

2. Write header-section

3. Write all entries into the file

4. Add the keyword "equation"

5. Write all connections into the file

6. Write end-section

\section{Figure 9: Writing the model file}

Confirmation dialogs inform the user about the CAEX file having been read in and the model file having been created successfully. For detailed information about the generation methodology see Beez (2007). The following figure summarizes the methodology graphically.

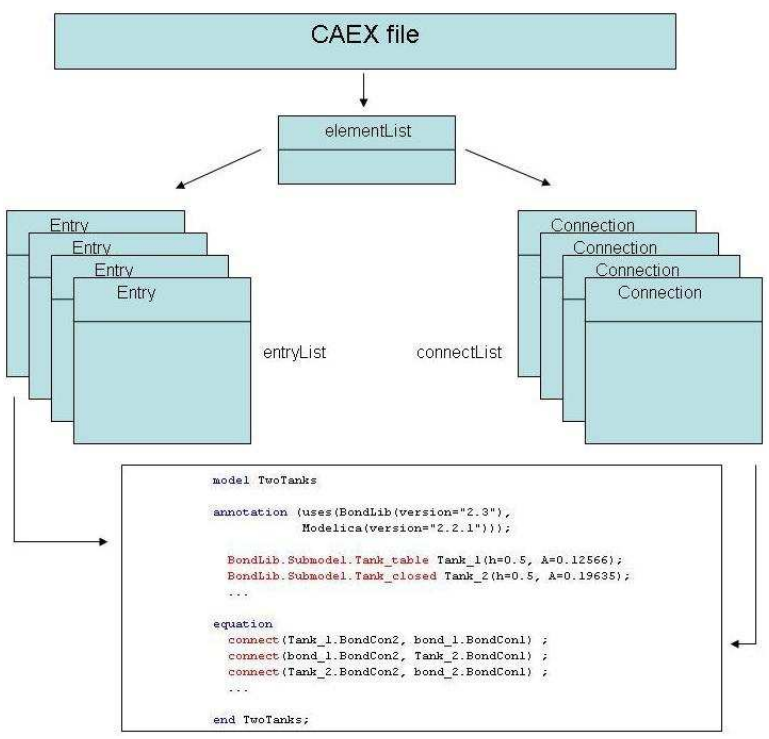

Figure 10: Generation methodology

\section{Validation example}

After an application is developed it needs to be tested and validated. Within this section the application has to prove that it can handle bondgraphic elements and that the developed methodology of creating models automatically functions. The aim is a model that can be compiled without errors and simulations can be run with it. In this section the pumped-storage power station build in Goldisthal, Germany, stands as the system the model is build from. Secondly, this section is to show that using these models can be useful for various purposes such as prediction, testing and verifying a controller-setup and many more.

Due to the ability of pumped-storage plants to produce a considerable amount of power within minutes and, therefore, giving the opportunity to react to load changes in the electricity network they are a welcome option. They are furthermore a cost-efficient way of storing energy during times of low demand.
That is why models of such system might be welcome for prediction or to test and verify controllers.

The structure, as described in the CAEX file used for the generation then, is as follows (according to [7]):

The upper basin (with a storage volume of 12 million $\mathrm{m}^{3}$ ) is connected to the turbine and the lower basin (with a storage volume of 18.9 million $\mathrm{m}^{3}$ ) via a pipe. The height difference between upper basin and turbine is approximately $350 \mathrm{~m}$, the maximum water height in the upper basin is $21 \mathrm{~m}$, in the lower basin it is $25 \mathrm{~m}$. Between the pipe and the turbine there is a valve to regulate the flow. The opening- and closingtime of the valve was assumed to be 30 seconds each. A pump between the lower basin and the second pipe leading into the upper basin is used to pump the water up again. Similar to the valve the pump needs 30 seconds to reach the maximum flow through the pump and another 30 seconds to shut down. The turbine is connected to a generator and the generator, itself, is the connected to the electricity net. A schematic of the system is given in the figure below.

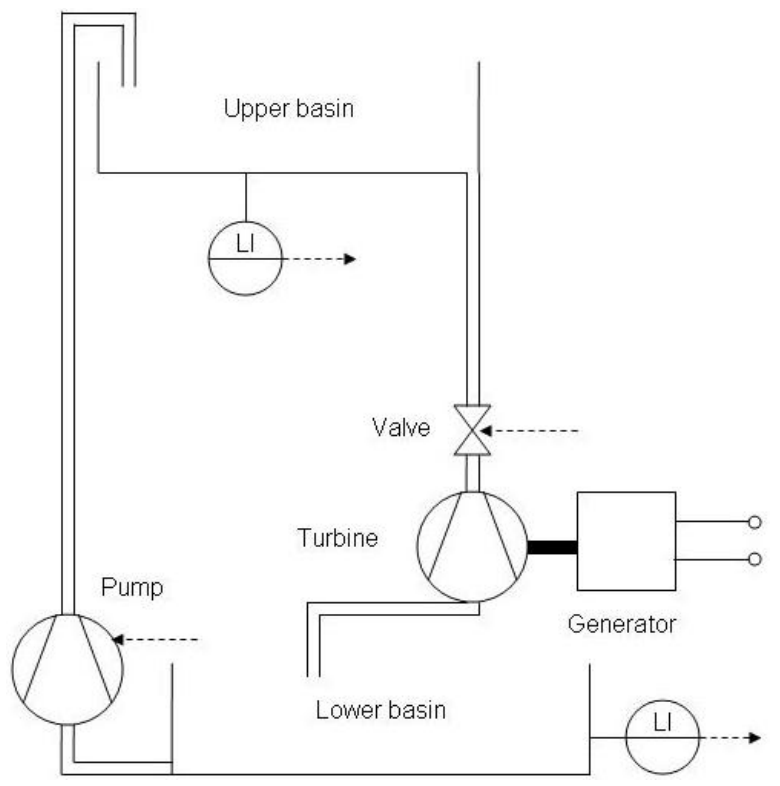

Figure 11: Schema of the power plant

After running the application on the CAEX file all the expected components are in the model. Using the internal error-checking routines in Dymola no errors occur. The following figure shows the Dymola/Modelica code that was generated by the application. 


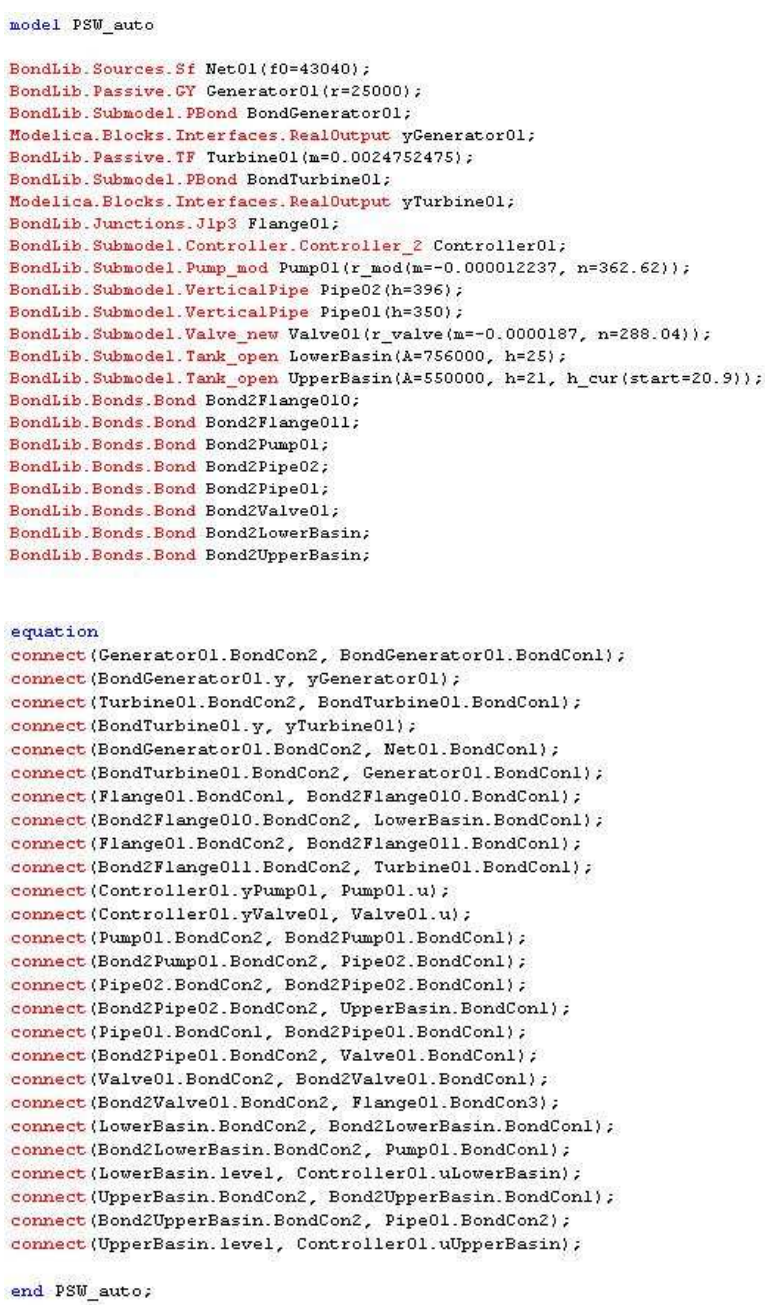

Figure 12: Generated model code

After making use of the possibility to add a startvalue for the height of water in the upper basin during the conversion and running a simulation in Dymola, the following figure shows a curve for the power output of the plant into the net in case the valve is completely opened.

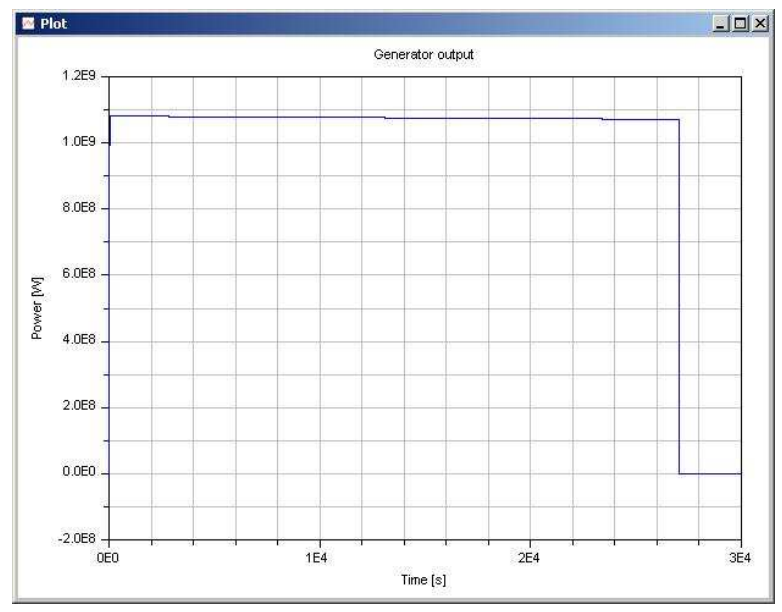

Figure 13: Simulation result for the generator
As expected, the power output slowly decreases from $1.08 \mathrm{GW}$ in the beginning to $1.07 \mathrm{GW}$ over a period of 7.5 hours. This is due to the lower water level in the upper basin in the end. This is another proof for the accuracy of the models. The time the upper basin needs to run dry is approximately the same as stated in [7].

The fact that no errors occur and the simulation is running as expected gives proof for the application's ability to handle the automatically generated. Replacing the simple controller used in this simulation with a controller that represents a real supply and demand-curve as shown in [8], the model can be used for various purposes as mentioned above.

\section{Conclusion}

The resulting program has proven able to create Bond Graph models out of CAEX files which match the given restrictions. Checking these models with the Dymola check-routine has showed that the models have no errors and that simulations can be run with them. Another important aspect is that the program code was written with an eye on future changes and further developments and, therefore, is maintained flexible.

As already mentioned possible uses for the application are various. The models generated could be used for testing and verifying controller set-ups, they could be implemented as simple models of systems used for prediction inside a Model Predictive Controller (MPC) or for simulations on systems in general.

On a long-term basis the models could be utilized for simulations on fault detection. However, this would require much more precision than and, thus, the development of precise component-models. Furthermore heat and multi-media models would have to be included. Another usage on a long-term basis might be visualizing the power flow within a system and, based on that, an optimization of the process itself or the architecture of a plant or system in general. For the purpose of visualizing the power flow the use of Power Bonds as explained in Beez (2008) can be most helpful.

\section{References}

[1] W3C 2007, World Wide Web Consortium, viewed 25 October, 2007, <www.w3.org>.

[2] R. Drath \& M. Fedai, "CAEX - a neutral data exchange format for engineering data", atp Automatisierungstechnische Praxis, Vol. 3, pp. 50-62, 2005. 
[3] D. C. Karnopp \& D. L. Margolis \& R. C. Rosenberg, System Dynamics: A Unified Approach, 2nd edn, John Wiley \& Sons, 1990.

[4] F. E. Cellier, Continuous System Modeling, SpringerVerlag, 1991.

[5] P. Fritzson, Principles of Object-Oriented Modeling and Simulation with Modelica 2.1, John Wiley \& Sons, 2004.

[6] S. Beez, Automatic generation of Bond Graph models out of CAEX: Requirements, possibilities and limitations, available via: Prof. Alexander Fay (alexander.fay@hsu-hh.de), Institut fuer Automatisierungstechnik, Helmut-Schmidt-University, Hamburg, 2007.

[7] Pumped Storage Power Plant Goldisthal Germany, Lahmeyer International, viewed 01 March, 2008, $<$ http://www.lahmeyer.de/e/units/ge/ps_ge1_e_200002 _goldisthal_2004_03.pdf>

[8] Wikipedia - The Free Encyclopedia 2008, Wikipedia Foundation, Inc., St. Petersburg, Florida, viewed 01 March, 2008, <http://de.wikipedia.org/wiki /Bild:Pumpspeicherkraftwerk.png>.

[9] S. Beez, Automatic Generation of Bond Graph Models of Process Plants, available via: Prof. Alexander Fay (alexander.fay@hsu-hh.de), Institut fuer Automatisierungstechnik, Helmut-Schmidt-University, Hamburg, 2008. 